\title{
Erasable diffractive grating couplers in silicon on insulator for wafer scale testing
}

\author{
R. Topley ${ }^{1}$, G. Martinez-Jimenez ${ }^{1}$, L. O'Faolain ${ }^{2}$, N. Healy ${ }^{1}$, S. Mailis ${ }^{1}$, D. J. Thomson ${ }^{1}$, F. Y. \\ Gardes $^{1}$, A. C. Peacock ${ }^{1}$, D. N. R. Payne ${ }^{3}$, G. Z. Mashanovich ${ }^{1}$, G. T. Reed ${ }^{1}$ \\ 1. Optoelectronics Research Centre, University of Southampton, UK \\ 2. SUPA, School of Physics and Astronomy, University of St. Andrews, UK \\ 3. Nano Research Group, ECS, University of Southampton, UK
}

\begin{abstract}
Test points are essential in allowing optical circuits on a wafer to be autonomously tested after selected manufacturing steps, hence allowing poor performance or device failures to be detected early and to be either repaired using direct write methods, or a cessation of further processing to reduce fabrication costs.

Grating couplers are a commonly used method for efficiently coupling light from an optical fibre to a silicon waveguide. They are relatively easy to fabricate and they allow light to be coupled into/out from any location on the device without the need for polishing, making them good candidates for an optical test point.

A fixed test point can be added for this purpose, although traditionally these grating devices are fabricated by etching the silicon waveguide, and hence this permanently adds loss and leads to a poor performing device when placed into use after testing. We demonstrate a similar device utilising a refractive index change induced by lattice disorder. Raman data collected suggests this lattice damage is reversible, allowing a laser to subsequently erase the grating coupler.
\end{abstract}

Keywords: Silicon photonics, diffraction gratings, grating couplers

\section{Introduction}

Integrated optical circuits are being investigated within several material platforms including silicon on insulator. Though silicon does not offer optimal optical characteristics, when compared to its rivals the platform does have a significant advantage in that the well-known manufacturing techniques can be adopted from silicon based microelectronics to photonic circuits. Silicon's history in electronics also has the allure of easier integration of electronics and photonics.

Unlike electronic integrated circuits, robust systems are not widely available for effectively autonomously testing optical integrated circuits at intermediate points on a waveguide, without introducing substantial losses into the devices. This makes quality control difficult as only the output of the entire optical circuit can be observed, providing information related to the complete optical circuit rather than localising a problem to the component level. A benefit of the Silicon on Insulator platform is its low cost; however processed silicon quickly becomes expensive. Each processing step costs money, and therefore testing is critical to reducing costs and increasing production yield. 
This work is not the first time autonomous testing methods have been considered. In 2008 a test probe was demonstrated, in which a metal grating coupler was fabricated directly onto a fibre facet [1]. The probe was designed to interact with the waveguide evanescent field, and the close proximity necessary for efficient coupling therefore requires a relatively flat circuit topology, and hence large protruding features in close proximity to the waveguide such as electrodes or an oxide cladding will prevent comprehensive circuit testing. A further risk exists as the grating was made using gold which may contaminate samples preventing subsequent processing in CMOS fabrication facilities, though other work on metal gratings fabricated directly onto the waveguide surface suggests gold may not be critical to the process [2]. A CMOS compatible concept for wafer scale testing was not presented until 2011. The method used erasable Bragg grating reflectors $[3,4]$ to reflect light travelling in the waveguide, such that the light can then be collected by using a circulator and detector arrangement connected to the circuit input. This offers an excellent wafer scale testing methodology for devices spanning a small wavelength range, with typical devices offering a few nanometres wavelength reflectivity, but broadband operation is problematic. The other disadvantage of this method is that monitoring reflection may not facilitate testing of the operation of devices which have asymmetrical transmission characteristics, such as modulators.

Here we demonstrate erasable technology for fabricating ion implanted diffractive grating couplers for use in wafer scale testing. We also provide analysis of the proposed erasing process using the analytical tool of Raman spectroscopy.

\section{Design \& simulation}

The implanted grating parameters were simulated and optimised using Lumerical's FDTD package [5]. Simulations suggested that the optical power extractable from a typical uniform surface relief grating coupler etched into a $220 \mathrm{~nm}$ thick silicon guiding layer is $\sim 50 \%$, which is in good agreement with other published literature [6]. Simulations comparable with those carried out for uniform surface relief grating couplers were performed for uniform ion implanted grating couplers. The results show a peak extracted power of approximately $45 \%$. The lower extracted power in the grating formed using ion implantation is consistent with the lower refractive index contrast in this case.

Surface relief grating couplers use a periodic reduction in the waveguides effective index, introduced by removing silicon material to leave a corrugated surface, which is typically filled in with a cladding material such as silicon dioxide. The localised refractive index change $(\Delta \mathrm{n})$ from silicon to air is $\Delta \mathrm{n}=-2.48$ or to a silicon dioxide cladding is $\Delta \mathrm{n}=-1.95$ which is relatively large by comparison to the localised refractive index change obtained in the case of ion implanted gratings formed in this work. Ion implanted gratings in this work have a periodic increase in refractive index caused by lattice disorder introduced into silicon by ion implantation with germanium which causes the formation of the amorphous allotrope of silicon. A maximum $\Delta \mathrm{n}$ of 0.585 has been demonstrated in the literature for germanium implantation into silicon [7]. It is also clear from the results, that $\Delta \mathrm{n}$ increases proportionately with an increase in the implantation dose condition. Ion implantation is performed through a mask to form periodic regions of amorphous and crystalline silicon, the amorphised regions exhibiting a positive $\Delta \mathrm{n}$ compared with crystalline silicon regions, raising the refractive index by $\Delta \mathrm{n} \approx 0.48$ for the doses used in this work. The simulations performed show that the efficiency reduction due to the reduced refractive index change is relatively small, with simulations suggesting that both methods of fabrication should offer similar performance characteristics when optimised, despite the smaller $|\Delta \mathrm{n}|$ for implanted devices.

Lattice disorder in silicon introduced by ion implantation and the corresponding refractive index changes were previously reported in, for example, [7-10]. The precise level of lattice disorder required for amorphous silicon formation varies in the literature (e.g. [11-13]) with a more detailed explanation given in [14]. However, there is a good agreement that when 
the defect density threshold for amorphisation is calculated with a defect density model, the crystalline - amorphous transition occurs with a critical point defect density of $1.15 \times 10^{22} \mathrm{~cm}^{-3}[12,15]$.

Simulation software packages, King (2D) and King 3D [16], have been shown to be in good agreement with the damage profiles observed by TEM imagery of similar implant designs to those considered here $[4,10]$. Both King packages share the same core method for calculating lattice damage, which is the Monte-Carlo binary collision approximation simulation method. A simulated damage level of $80 \%$ lattice disorder is considered amorphisation, which will reach the desired refractive index change of $\sim 0.5$. The King software package can quickly and efficiently simulate the depth and degree of lattice disorder. Optical simulations show that optimal performance is achieved with an amorphous depth of $\sim 130 \mathrm{~nm}$, depending on the specific wavelength [17]. King simulations show that an amorphous thickness of $131 \mathrm{~nm}$ is achieved for a dose of $1 \times 10^{15}$ ions $\mathrm{cm}^{-2}$ with an implant energy of $100 \mathrm{keV}$, and this dose has previously demonstrated successful amorphisation $[4,10]$. King 3D can expand on this information with a more lengthy simulation to show the full disorder profile, including lateral data which can be critical to the device design. The full implant profile for a 100keV King 3D simulation with a dose of $1 \times 10^{15}$ ions $\mathrm{cm}^{-2}$ is shown in Figure 1.

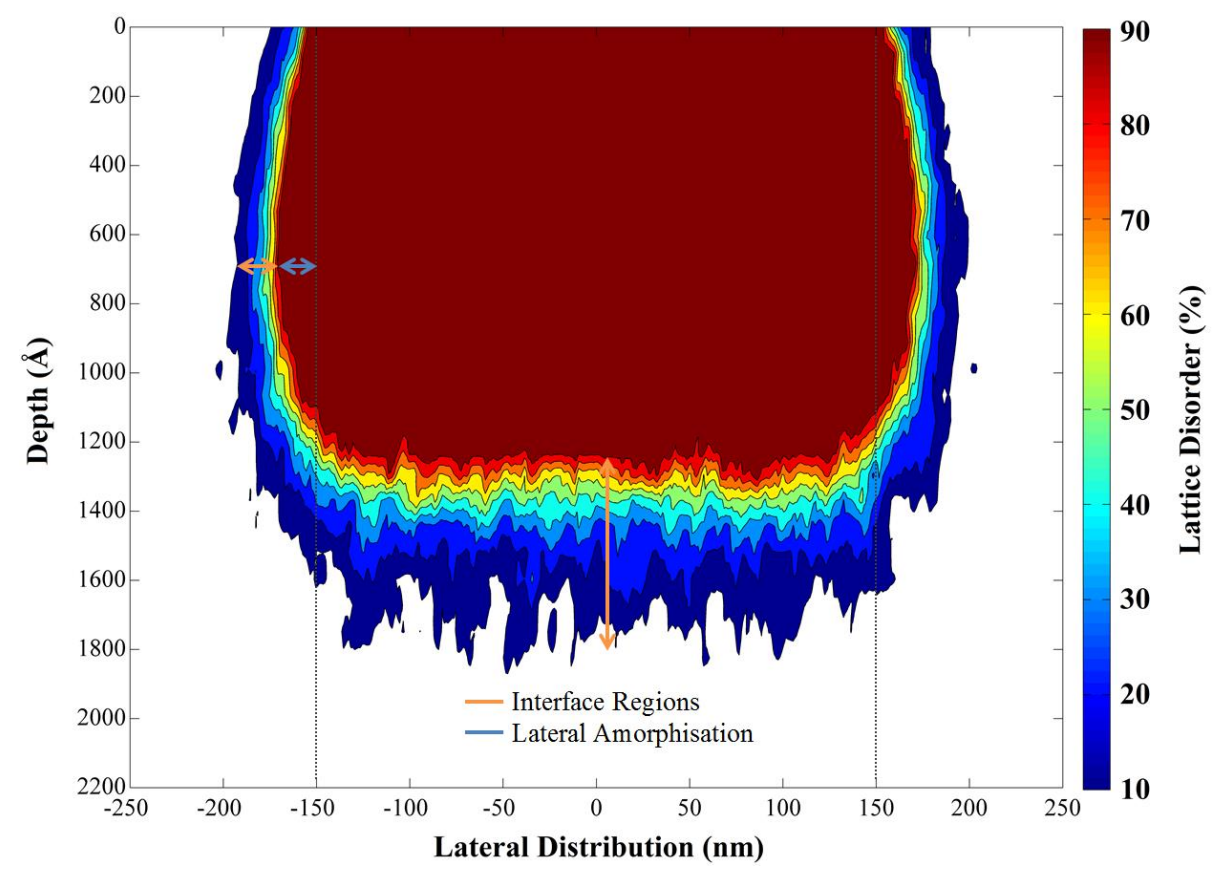

Figure 1 - Amorphisation profile for $100 \mathrm{keV}$ energy, $1 \times 10^{15}$ ions $\mathrm{cm}^{-2}$, implanted through a 300nm slit. Dotted lines represent the width of the mask used.

The disadvantage of the Monte-Carlo binary collision approximation method for simulation, is that it does not account for dynamic annealing or the transport of defects [18], hence the simulation must be considered to be most accurate for low temperature, liquid nitrogen cooled implantation. Large interface regions between the amorphous and crystalline silicon are apparent in the King 3D simulation shown in Figure 1, and the interface regions are neither fully amorphous nor fully crystalline. Implanting at room temperature however, can significantly reduce the size of these interface regions. When the implant is performed at room temperature these regions are not visible, this is due to dynamic annealing effects which occur during implantation [18], as verified via TEM images of similar implant designs $[4,10]$. The implant time, and hence the cost, are also reduced by using a room temperature implantation as the system does not have the delay of 
prior cooling or the subsequent warming, which is required after implantation. The King 3D simulation does show $22 \mathrm{~nm}$ of lateral amorphisation above the $80 \%$ damage threshold, which will alter the effective duty cycle of the implanted devices compared with the mask template, and consequently the optical performance may not exactly match the simulations. Implanting a $600 \mathrm{~nm}$ grating with a mask duty cycle of 0.5 , based on the King 3D simulation, would result in a 0.54 effective duty cycle. This does not reduce optical efficiency significantly in simulations. However, an apodised grating coupler would see much more significant effects from the duty cycle variation due to the smaller feature sizes typically involved with this style of device.

\section{Experimental Results}

The thickness and refractive index profile of the ion implanted silicon was measured on a bulk implanted substrate using ellipsometry, 6 different angles of incidence were tested to ensure an accurate result. The data collected was amalgamated and analysed using the CompleteEASE® software package [19] and subsequently fitted using a B-Spline numerical analysis. The discrepancy between simulated and measured data was below $3 \mathrm{~nm}$, which showed the amorphised silicon layer thickness to be in good agreement with simulated data. The refractive index of the amorphous layer at a wavelength of $1.55 \mu \mathrm{m}$ was found to be 3.96 yielding a $\Delta \mathrm{n}$ of 0.48 , which is in good agreement with the expected $\Delta \mathrm{n}$ of the order of up to 0.585 from other literature $[7,10,20]$.

Raman spectroscopy has also been performed on the silicon regions before and after laser annealing to assess material composition and the crystallinity of regrown material as shown in Figure 2. A 488nm, CW laser was used at $180 \mathrm{~mW}$ for the annealing tests, the laser spot was measured to be $\sim 1.5 \mu \mathrm{m}$ with a scan rate of $10 \mu \mathrm{m} / \mathrm{s}$. Comparing the measured Raman spectra with characterisation data obtained for amorphous semiconductors from [21], confirms the successful formation of amorphous silicon prior to annealing, highlighted by broad peaks at $140 \mathrm{~cm}^{-1}$ and $480 \mathrm{~cm}^{-1}$. Using the same characterisation data it is also important to highlight an absence of peaks at $75 \mathrm{~cm}^{-1}$ or in the vicinity of $270 \mathrm{~cm}^{-1}$ which shows the material is not significantly impacted by the choice of germanium for amorphisation. A sharp peak is also observed at $520 \mathrm{~cm}^{-1}$, both before and after annealing, this shows a contribution from crystalline silicon. A crystalline peak is observed in the amorphous data is in part due to the Raman laser spot size being slightly larger than the width of an amorphous grating period region $(300 \mathrm{~nm})$, and also as the $633 \mathrm{~nm}$ light used for Raman spectroscopy penetrates into the crystalline silicon waveguide beneath the implanted region.

Interrogating the same grating region with Raman spectroscopy post laser annealing shows clearly that only a single peak at $520 \mathrm{~cm}^{-1}$ exists. This demonstrates the amorphous regions have been successfully annealed. A Voigt fit applied to the spectrum taken on the annealed material reveals a Lorentzian linewidth of $4.3 \mathrm{~cm}^{-1}$ compared to $2.7 \mathrm{~cm}^{-1}$ for single crystal silicon. This suggests the annealed silicon is in fact polycrystalline, and a reduction in material quality may account for some of the residual losses. It is likely that the annealing process can be further optimised to improve the annealed silicon's crystallinity and hence reduce this loss in future devices. 


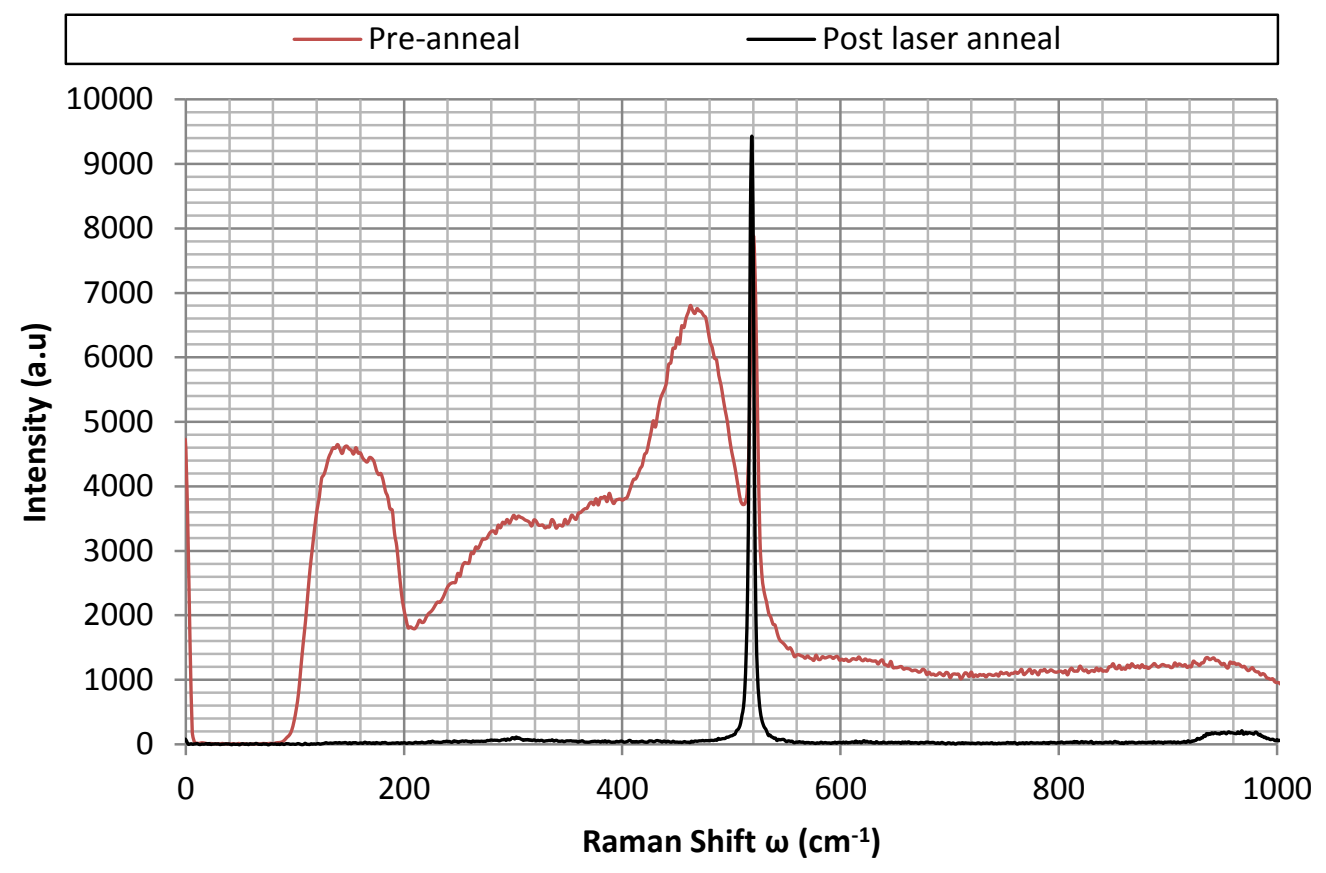

Figure 2 - Raman data for amorphous silicon before and after annealing.

Five gratings were fabricated with periods of 580, 590, 600, 610 and 620nm all with the same 0.5 resist mask duty cycle. To reduce back reflections during measurement, the implanted couplers were measured at a $17^{\circ}$ angle of incidence. Etched grating couplers were also designed and fabricated, the waveguide and taper designs for both devices are identical to ensure congruency between the implanted and etched structures for comparison. A period of 700nm and an etch depth of $70 \mathrm{~nm}$ was used for the etched devices. The surface relief grating coupler and taper combination exhibited a coupling loss of $4.5 \mathrm{~dB}$ in the best device which is comparable to the best amorphous silicon grating coupler with taper structure result of $5.5 \mathrm{~dB}$, as shown in Fig 3 .

The implanted devices show a $1 \mathrm{~dB}$ and $3 \mathrm{~dB}$ bandwidth of $32 \mathrm{~nm}$ and $56 \mathrm{~nm}$ respectively. The etched grating couplers fabricated concurrently with the implanted devices show analogous $1 \mathrm{~dB}$ and $3 \mathrm{~dB}$ bandwidths of $30 \mathrm{~nm}$ and $51 \mathrm{~nm}$ respectively, this performance is comparable to that of uniform etched gratings published in the literature [22, 23]. 


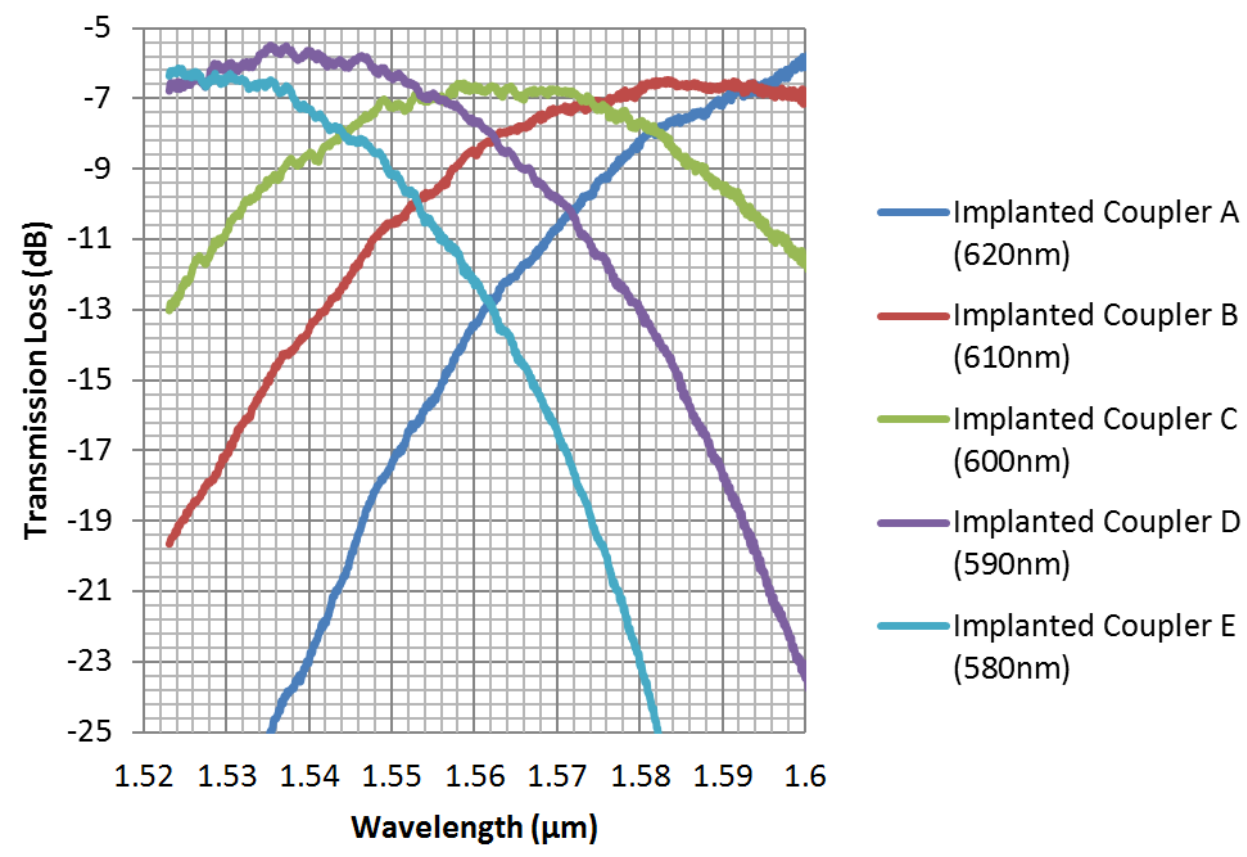

Fig 3 - Performance of implanted grating couplers at different central wavelengths

As expected the implanted structures perform comparably with etched gratings. The silicon to air, or silicon to silicon dioxide $\Delta \mathrm{n}$ of 2.48 and 1.95 respectively is considerably larger than the $\Delta \mathrm{n}$ of 0.48 introduced by the amorphous to crystalline silicon interface. A lower index contrast is expected to reduce coupling strength, yet empirically the performance is only $1 \mathrm{~dB}$ lower for the amorphous grating coupler compared with the implanted coupler.

\section{Conclusions}

A novel method of fabrication for diffractive grating couplers has been demonstrated in this work, the effective index modulation has been introduced via ion implantation to form an amorphous allotrope of silicon using lattice disorder. This method allows the surface planarity of the silicon waveguide devices to be maintained whilst still providing comparable coupling efficiency to an etched grating coupler for coupling between a silicon wire waveguide and a single mode optical fibre.

The coupling loss for an implanted grating and taper combination shows a performance reduction of only $1 \mathrm{~dB}$ compared with its etched counterpart and the $1 \mathrm{~dB}$ and $3 \mathrm{~dB}$ bandwidths are also shown to be comparable to those of surface relief gratings.

Furthermore, Raman data shows the transition from amorphous to polycrystalline silicon is possible for these devices using laser annealing techniques, which may allow the fabricated devices to be erased in a simple and controlled manner.

\section{Acknowledgments}

The authors would like to thank EPSRC, UK for funding this work. Goran Mashanovich would like to acknowledge support from the Royal Society. 


\section{References}

[1] S. Scheerlinck, D. Taillaert, D. Van Thourhout et al., "Flexible metal grating based optical fiber probe for photonic integrated circuits," Applied Physics Letters, 92(3), 031104-3 (2008).

[2] S. Scheerlinck, J. Schrauwen, F. Van Laere et al., "Efficient, broadband and compact metal grating couplers for silicon-on-insulator waveguides," Optics Express, 15(15), 9625-9630 (2007).

[3] R. Loiacono, G. T. Reed, G. Z. Mashanovich et al., "Low-energy silicon-on-insulator ion implanted gratings for optical wafer scale testing." 7943, 794310-794310-6.

[4] R. Loiacono, R. Topley, A. Nakyobe et al., "Very low energy implanted Bragg gratings in SOI for wafer scale testing applications." 51-53.

[5] Lumerical, "FDTD Solutions 8.7."

[6] C. Kopp, and A. Chelnokov, "Fiber grating couplers for silicon nanophotonic circuits: Design modeling methodology and fabrication tolerances," Optics Communications, 282(21), 4242-4248 (2009).

[7] K. F. Heidemann, "Complex-refractive-index profiles of $4 \mathrm{MeV} \mathrm{Ge}$ ion-irradiation damage in silicon," Philosophical Magazine Part B, 44(4), 465-485 (1981).

[8] E. Baranova, V. Gusev, W. Martynenko et al., "On silicon amorphization during different mass ion implantation," Radiation Effects, 18(1-2), 21-26 (1973).

[9] R. Loiacono, G. T. Reed, R. Gwilliam et al., [Germanium implanted Bragg gratings in silicon on insulator waveguides] SPIE, (2010).

[10] R. Loiacono, G. T. Reed, G. Z. Mashanovich et al., "Laser erasable implanted gratings for integrated silicon photonics," Opt. Express, 19(11), 10728-10734 (2011).

[11] L. A. Christel, J. F. Gibbons, and T. W. Sigmon, "Displacement criterion for amorphization of silicon during ion implantation," Journal of Applied Physics, 52(12), 7143-7146 (1981).

[12] H. Cerva, and G. Hobler, "Comparison of Transmission Electron Microscope Cross Sections of Amorphous Regions in Ion Implanted Silicon with Point-Defect Density Calculations," Journal of The Electrochemical Society, 139(12), 3631-3638 (1992).

[13] J. R. Dennis, and E. B. Hale, "Crystalline to amorphous transformation in ion-implanted silicon: a composite model," Journal of Applied Physics, 49(3), 1119-1127 (1978).

[14] G. Hobler, and G. Otto, "Status and open problems in modeling of as-implanted damage in silicon," Materials Science in Semiconductor Processing, 6(1-3), 1-14 (2003).

[15] O. Engström, S. Bengtsson, G. I. Andersson et al., "Electrical characterization of bonding interfaces," Journal of The Electrochemical Society, 139(12), 3638-3644 (1992).

[16] G. Lulli, M. Bianconi, A. Parisini et al., "Structural characterization and modeling of damage accumulation in In implanted Si," Journal of Applied Physics, 95(1), 150-155 (2004).

[17] R. Topley, L. O'Faolain, D. J. Thomson et al., "Planar surface implanted diffractive grating couplers in SOI," Optics Express, 22(1), 1077-1084 (2014).

[18] G. F. Cembali, P. G. Merli, and F. Zignani, "Self-annealing of ion-implanted silicon: First experimental results," Applied Physics Letters, 38(10), 808-810 (1981).

[19] I. Ja.A. Woollam Co. "http://www.jawoollam.com/completeease.html", access year: 2013

[20] A. P. Knights, K. J. Dudeck, W. D. Walters et al., "Modification of silicon waveguide structures using ion implantation induced defects," Applied Surface Science, 255(1), 75-77 (2008).

[21] D. D. Allred, J. Gonzalez-Hernandez, O. V. Nguyen et al., "Raman scattering and x-ray diffraction characterization of amorphous semiconductor multilayer interfaces," Journal of Materials Research, 1(03), 468475 (1986).

[22] D. Taillaert, P. Bienstman, and R. Baets, "Compact efficient broadband grating coupler for silicon-on-insulator waveguides,” Opt. Lett., 29(23), 2749-2751 (2004). 
[23] S. K. Selvaraja, D. Vermeulen, M. Schaekers et al., "Highly efficient grating coupler between optical fiber and silicon photonic circuit." 1-2. 